\title{
Computational Analysis for Morphological Evolution in Pyrolysis for Micro/Nanofabrication
}

\author{
Myeongseok Yang, ${ }^{1}$ Wooju Lee, ${ }^{1}$ Heungjoo Shin, ${ }^{2}$ and Dongchoul Kim ${ }^{1}$ \\ ${ }^{1}$ Department of Mechanical Engineering, Sogang University, Seoul 121-742, Republic of Korea \\ ${ }^{2}$ School of Mechanical and Advanced Materials Engineering, Ulsan National Institute of Science and Technology (UNIST), \\ Ulsan 689-798, Republic of Korea
}

Correspondence should be addressed to Dongchoul Kim; dckim@sogang.ac.kr

Received 19 February 2015; Accepted 24 June 2015

Academic Editor: Hui Zeng

Copyright (C) 2015 Myeongseok Yang et al. This is an open access article distributed under the Creative Commons Attribution License, which permits unrestricted use, distribution, and reproduction in any medium, provided the original work is properly cited.

Pyrolysis is recently proposed as an efficient fabrication technique of micro/nanoscale carbon structures. In order to understand the morphological evolution in pyrolysis and design the final shape of carbon structure, this study proposes a comprehensive model that incorporates the essential mechanisms of pyrolysis based on the phase field framework. Computational analysis with the developed model provides information about the effect of interface energy and kinetic rate on the morphological evolution in pyrolysis.

\section{Introduction}

During the pyrolysis process, various intermediate materials are generated from carbonaceous precursor, and a carbon structure remains after sufficient process time. This characteristic of pyrolysis has been applied to biofuel production $[1,2]$. Pyrolysis has been also suggested as an efficient manufacturing technique for micro/nanostructures with glassy carbon. As an electrode material, glassy carbon is widely used due to its remarkable mechanical and electronic properties with chemical stability at low cost. However, due to the high brittleness of glassy carbon, it is very difficult to utilize glassy carbon for micro/nanodevices even with a simple geometry. Recently, a fabrication of carbon-microelectromechanical system (C-MEMS) is suggested as a plausible technique that can overcome the limit of brittle glassy carbon by pyrolyzing prepatterned precursors using UV-lithography [3], e-beam lithography [4], nanotemplate [5], or electrospinning [6]. In order to develop a solid technique for micro/nanofabrication with pyrolysis, an efficient and reliable computational model needs to be developed.

Since the theoretical analysis of the pyrolysis of wood was conducted, theoretical studies of the pyrolysis have been performed [7-11]. The pyrolysis process of biomass such as cellulose was analyzed by an approach based on a chemical kinetic reaction $[7,12]$. Although the early models considered a simplified one-step reaction, the multistep reaction process was recently incorporated into a kinetic model $[9,10]$. The kinetic model described the successive reaction and the mass of reactants and products were quantitatively estimated in the reaction $[10,13,14]$. However, the morphological evolution such as a shrinking process in the pyrolysis is difficult to be described by kinetic model because the model did not incorporate any interfacial effects, interface, and surface energies of each component, which significantly affect the morphological evolution of micro- and nanostructures [15]. In utilizing the pyrolysis for the carbon nanofabrication, the dimensional analysis of morphological evolution in the pyrolysis is an important prerequisite.

Therefore, we propose a three-dimensional dynamic model for pyrolysis. The evolution of each component such as polymer precursor and carbon, which is driven by multiple mechanisms, is systematically modeled. The model incorporates thermal decomposition, diffusion of components, interfacial energies between components, and surface energies of components. To efficiently simulate the dynamic evolution of 


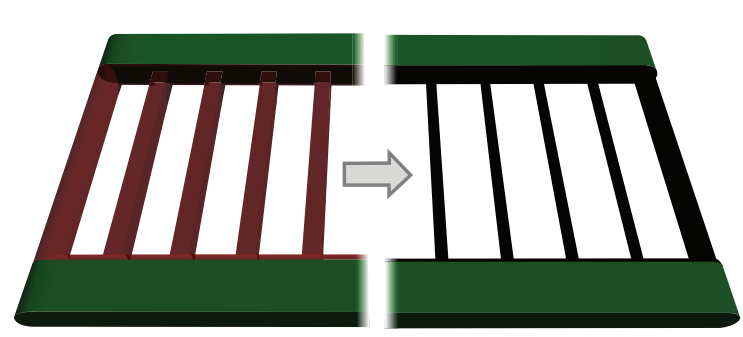

(a)

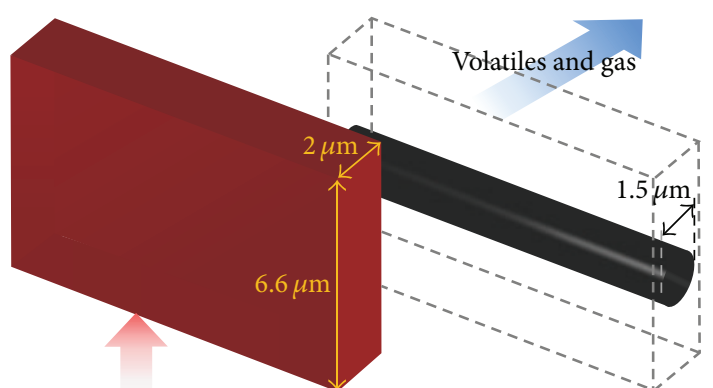

Thermal energy

Volume shrinkage
Polymer precursor
$c_{1}$ (polymer) $\begin{gathered}\text { Carbon structure } \\ c_{2} \text { (carbon) }\end{gathered}$

(b)

FIGURE 1: (a) Schematic drawing of pyrolysis process for generation of a carbon nanostructure from polymer precursor. (b) Model for pyrolysis process describing the decomposition and generation of components.

decomposed materials in pyrolysis, a diffusive interface approach is employed with the kinetic model. The diffusive interface approach has demonstrated high reliability in simulating complicated morphological evolutions such as the phase separation, solidification, and self-assembly of various microand nanoscale structures [16-18]. With the developed model, simulations are performed analyzing the characteristics of pyrolysis with respect to the morphology of carbon structure.

\section{Computational Methods}

In the pyrolysis process, biomaterials at high temperature are transferred to volatile organic compound, gas, and carbon by thermal energy, and eventually a carbon structure remains. We develop a multiphysics and multicomponent model based on the phase field approach. As shown in Figure 1, we define a volume faction $c_{1}(x, y, z, t)$ by polymer and $c_{2}(x, y, z, t)$ by carbon. The volatile and gas are defined by $c_{3}(x, y, z, t)$, that is, $1-c_{1}(x, y, z, t)-c_{2}(x, y, z, t)$. In the pyrolysis process, an oven is heated until the pyrolysis temperature and the temperature are maintained. Thus, we consider an isothermal model.

2.1. Morphological Evolution. The evolution of each component is induced by the minimizing process of the total free energy of the system. We define free energy $\mathscr{F}$ as quoted by the Cahn-Hilliard model [19]:

$$
\begin{aligned}
\mathscr{F} & =\int f\left(c_{1}, c_{2}, c_{3}\right)+h_{11}\left(\nabla c_{1}\right)^{2}+h_{22}\left(\nabla c_{2}\right)^{2} \\
& +h_{12}\left(\nabla c_{1} \cdot \nabla c_{2}\right) d V .
\end{aligned}
$$

Because $c_{3}$ is volatile or gas, the interactions of $c_{3}$ and other materials can be ignored. The first term, $f\left(c_{1}, c_{2}, c_{3}\right)$, is the volumetric local free energy of materials. All of gradient terms are the interfacial energies between materials. Each interfacial energy coefficient is $h_{11}, h_{22}$, and $h_{12}$. The local free energy generally represents a double well potential. The local free energy of three components has been derived from the studies of two-phase solidification [20]. Here, we assume the local free energy to consist of the linear combination of double well potentials of components, which have the local minimum points at $c_{i}=1$ and $c_{i}=0$ :

$$
\begin{aligned}
& f\left(c_{1}, c_{2}, c_{3}\right)=f_{0} c_{1}^{2}\left(1-c_{1}\right)^{2}+f_{0} c_{2}^{2}\left(1-c_{2}\right)^{2}+f_{0} c_{3}^{2}(1 \\
& \left.\quad-c_{3}\right)^{2}+\frac{a_{1}}{4} c_{1}^{2}\left[15\left(1-c_{1}\right)\left\{1+c_{1}-\left(c_{3}-c_{2}\right)^{2}\right\}\right. \\
& \left.\quad+c_{1}\left(9 c_{1}^{2}-5\right)\right]+\frac{a_{2}}{4} \\
& \quad \cdot c_{2}^{2}\left[15\left(1-c_{2}\right)\left\{1+c_{2}-\left(c_{1}-c_{3}\right)^{2}\right\}\right. \\
& \left.\quad+c_{2}\left(9 c_{2}^{2}-5\right)\right]+\frac{a_{3}}{4} \\
& \quad \cdot c_{3}^{2}\left[15\left(1-c_{3}\right)\left\{1+c_{3}-\left(c_{2}-c_{1}\right)^{2}\right\}\right. \\
& \left.\quad+c_{3}\left(9 c_{3}^{2}-5\right)\right] .
\end{aligned}
$$

The higher order terms of $a_{i}$ are reduced by taking $a_{1}=$ $a_{2}=a_{3}$. Then, local free energy $f\left(c_{1}, c_{2}, c_{3}\right)$ is simplified by

$$
\begin{aligned}
f\left(c_{1}, c_{2}, c_{3}\right)= & f_{0} c_{1}^{2}\left(1-c_{1}\right)^{2}+f_{0} c_{2}^{2}\left(1-c_{2}\right)^{2} \\
& +f_{0} c_{3}^{2}\left(1-c_{3}\right)^{2}+1 .
\end{aligned}
$$

The evolution of a free energy system is driven by chemical potential that can be expressed in the model by $\mu_{i}=$ $d \mathscr{F} / d c_{i}$. It is related to the driving forces that could be represented by $\mathbf{F}_{d}=-\nabla \cdot \mu_{i}$. The flux is represented as $J_{i}=-M_{i} \nabla \mu_{i}$, where $M_{i}$ is mobility of material. The diffusive interface is described by $P(x)=x^{3}\left(6 x^{3}-15 x^{2}+10\right)$. With the mass conservation relation, $\partial c_{1} / \partial t=\nabla \cdot J_{1}$, and $\partial c_{2} / \partial t=\nabla \cdot J_{2}$, the expressions of $c_{1}$ and $c_{2}$ are obtained as follows:

$$
\begin{aligned}
& \frac{\partial c_{1}}{\partial t}=\nabla \cdot\left[M_{1} P\left(c_{1}\right) \nabla \mu_{1}\right], \\
& \frac{\partial c_{2}}{\partial t}=\nabla \cdot\left[M_{2} P\left(c_{2}\right) \nabla \mu_{2}\right],
\end{aligned}
$$


where

$$
\begin{aligned}
\mu_{1}(T)= & \frac{\partial}{\partial c_{1}}\left\{\sum_{i=1}^{3} f_{0} c_{i}^{2}\left(1-c_{i}\right)^{2}\right\}-2 h_{11} \nabla^{2} c_{1} \\
& -h_{12} \nabla^{2} c_{2}, \\
\mu_{2}(T)= & \frac{\partial}{\partial c_{2}}\left\{\sum_{i=1}^{3} f_{0} c_{i}^{2}\left(1-c_{i}\right)^{2}\right\}-2 h_{22} \nabla^{2} c_{2} \\
& -h_{12} \nabla^{2} c_{1} .
\end{aligned}
$$

The chemical potentials indicate the dynamic evolution of structure to minimize the bulk and interfacial energies.

2.2. Thermal Decomposition. In the pyrolysis process, the polymer is gradually decomposed by thermal energy, which has been described with a kinetic model $[10,13,15,21]$. Incorporating the kinetic model, the thermal decomposed polymer, $c_{1}$, and transferred carbon, $c_{2}$, are described by

$$
\begin{aligned}
& \frac{\partial c_{1}}{\partial t}=-\nu c_{1}, \\
& \frac{\partial c_{2}}{\partial t}=\nu \theta c_{1},
\end{aligned}
$$

where $\nu$ is the reaction rate at isothermal and $\theta$ is the generation rate of $c_{2}$ from the decomposed $c_{1}$. By combining (4) and (6), the expression of $c_{1}$ and $c_{2}$ is obtained as follows:

$$
\begin{aligned}
& \frac{\partial c_{1}}{\partial t}=\nabla \cdot\left[M_{1} P\left(c_{1}\right) \nabla \mu_{1}\right]-v c_{1}, \\
& \frac{\partial c_{2}}{\partial t}=\nabla \cdot\left[M_{2} P\left(c_{2}\right) \nabla \mu_{2}\right]+\nu \theta c_{1} .
\end{aligned}
$$

In this pyrolysis process, the decomposed polymer generates amorphous carbon. All these materials dynamically change their component ratio to minimize the total free energy and evolve into nanoscale structures.

2.3. Numerical Computation. By employing the characteristic time $\tau$ and length $L$, we obtain dimensionless numbers; $\tau=$ $L^{2} / M_{1} f_{0}, \bar{h}_{11}=2 h_{11} / L^{2} f_{0}, \bar{h}_{22}=2 h_{22} / L^{2} f_{0}, \bar{h}_{12}=2 h_{12} / L^{2} f_{0}$, and $\eta=M_{2} / M_{1}$. From normalization, the dimensionless equations are derived as follows:

$$
\begin{aligned}
\frac{\partial c_{1}}{\partial t} & =\nabla \cdot\left[P\left(c_{1}\right) \nabla \bar{\mu}_{1}\right]-v \tau c_{1}, \\
\frac{\partial c_{2}}{\partial t} & =\nabla \cdot\left[\eta P\left(c_{2}\right) \nabla \bar{\mu}_{2}\right]+v \tau \theta c_{1}, \\
\bar{\mu}_{1} & =\frac{\partial}{\partial c_{1}}\left\{\sum_{i=1}^{3} \bar{f}_{0} c_{i}^{2}\left(1-c_{i}\right)^{2}\right\}-\bar{h}_{11} \nabla^{2} c_{1}-\frac{\bar{h}_{12}}{2} \nabla^{2} c_{2}, \\
\bar{\mu}_{2} & =\frac{\partial}{\partial c_{2}}\left\{\sum_{i=1}^{3} \bar{f}_{0} c_{i}^{2}\left(1-c_{i}\right)^{2}\right\}-\bar{h}_{22} \nabla^{2} c_{2}-\frac{\bar{h}_{12}}{2} \nabla^{2} c_{1} .
\end{aligned}
$$

$c_{1}$ and $c_{2}$ represent the change of polymer and carbon volume fraction, respectively.

\section{Results and Discussion}

In order to understand the morphological evolution of pyrolysis process, a series of simulations have been performed. A microscale bar with dimension of $2.0 \mu \mathrm{m} \times 6.6 \mu \mathrm{m}$ is considered. The ratio of mobility between polymer and carbon is assumed to be 0.5 . The generation rate of carbon from the decomposed polymer, $\theta$, is set to 0.2 .

3.1. Evolution of Polymer and Carbon in Pyrolysis. During the pyrolysis process, a polymer precursor is gradually decomposed by heat and eventually carbon remained. The volatiles are vaporized and the total volume is decreased. As shown in Figure 2(a), the simulation result describes the morphological evolution during the pyrolysis, which cannot be observed in experiments. The polymer and generated carbon diffuse and agglomerate to minimize the surface energy. Eventually, a microscale polymer bar decomposes and becomes a reduced carbon structure whose diameter is $1.5 \mu \mathrm{m}$. The volume of polymer is reduced below $10 \%$ after 4,000 5,000 steps. About $90 \%$ of polymer precursors are decomposed into carbon and volatiles within 5,000 steps. The reaction rate decreases as time passes since the polymer reactant is diminished by reaction. When the remaining polymer becomes about $10 \%$, the reaction rate is significantly decreased as shown in Figure 2(b).

3.2. Effect of Surface Interaction. In order to investigate the effect of the surface energy and interface energy of polymer and carbon on the morphological evolution during the pyrolysis, simulations with different combination of $h_{11}, h_{22}$, and $h_{12}$ are performed as shown in Figure 3. When the interface energy between polymer and carbon is the same as or smaller than the surface energy of polymer and carbon, a well-defined single bar of carbon is generated at the final stage. There are, however, different morphological evolutions during pyrolysis. When the surface energy of polymer is twice larger than the interface energy and the surface energy of carbon, the polymer has strong self-interaction and shows more spherical cross section than that of case when it is the same as other energies at 5,000 steps as shown in Figure 3(b). When the surface energy of carbon is twice larger than the interface energy and the surface energy of polymer, the carbon already forms linkage at 5,000 steps rather than forming four bars surrounding the polymer structure as shown in Figure 3(c).

The effect of the interface energy between polymer and carbon is presented in Figures 3(d) and 3(e). Case with the interface energy being smaller than other surface energies shows similar evolutions with cases with the interface energy being the same as other surface energies as shown in Figure 3(d). However, when the interface energy is larger than other surface energies, a discrete assembly in the longer direction of cross section is generated. According to the free energy minimization, the driving force to minimize the interface energy reduces the interface area between polymer and carbon. It leads to a rapid separation of two components. As a result, the separation of polymer and carbon is faster than other cases and discrete multiple carbon structures can be 

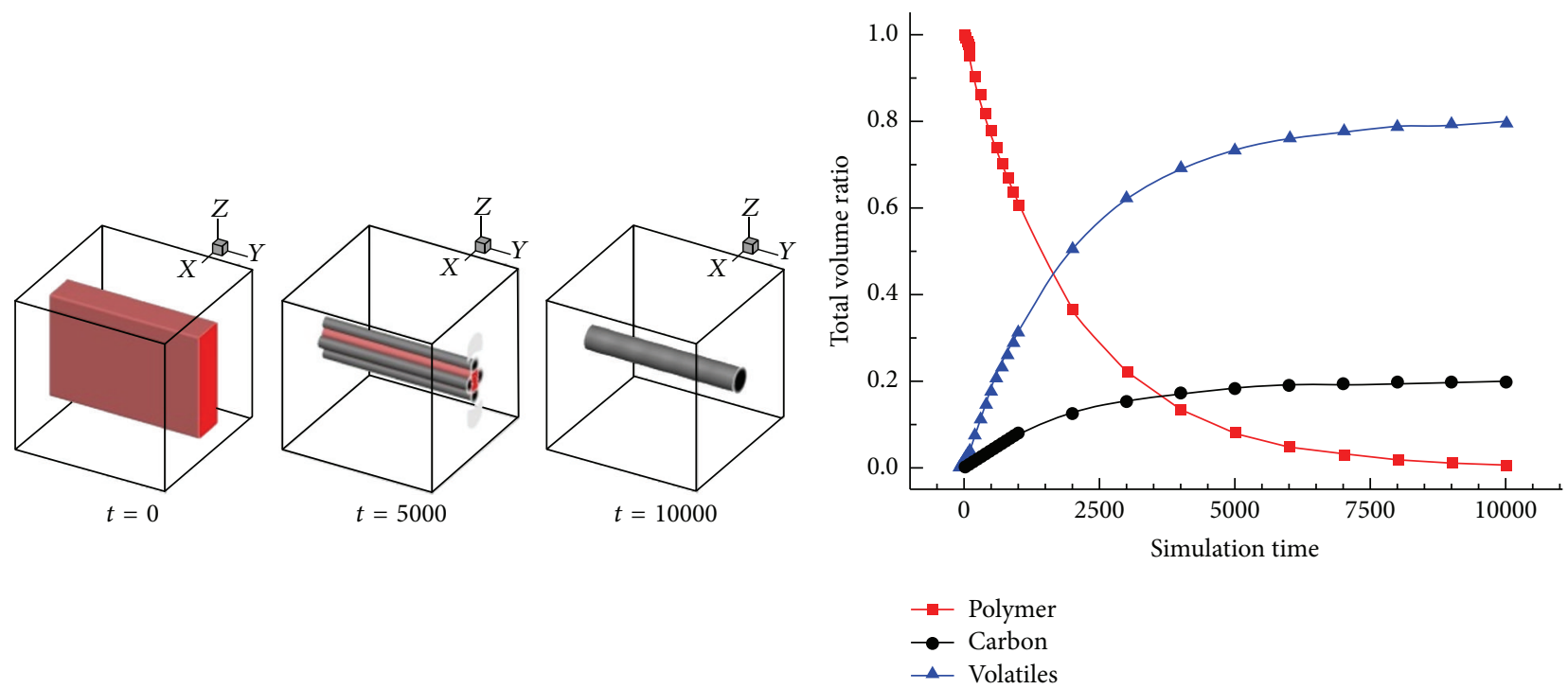

(a)

(b)

Figure 2: (a) Simulated pyrolysis process in isothermal system. Polymer and carbon are indicated by red and black colors, respectively. (b) Polymer diminution and carbon generation with respect to simulation time. Polymer, carbon, and volatiles are indicated by red, black, and blue, respectively.

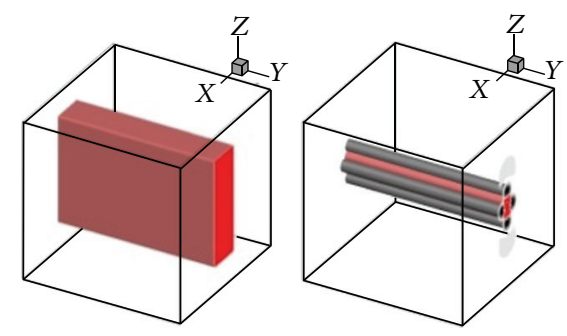

(a)
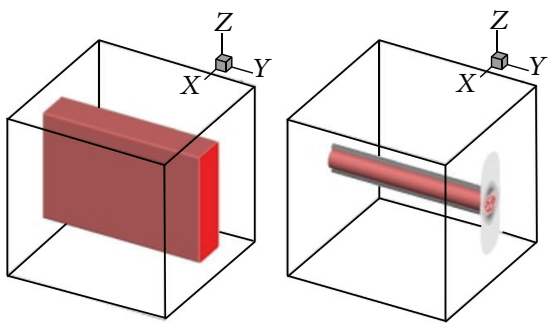

(c)
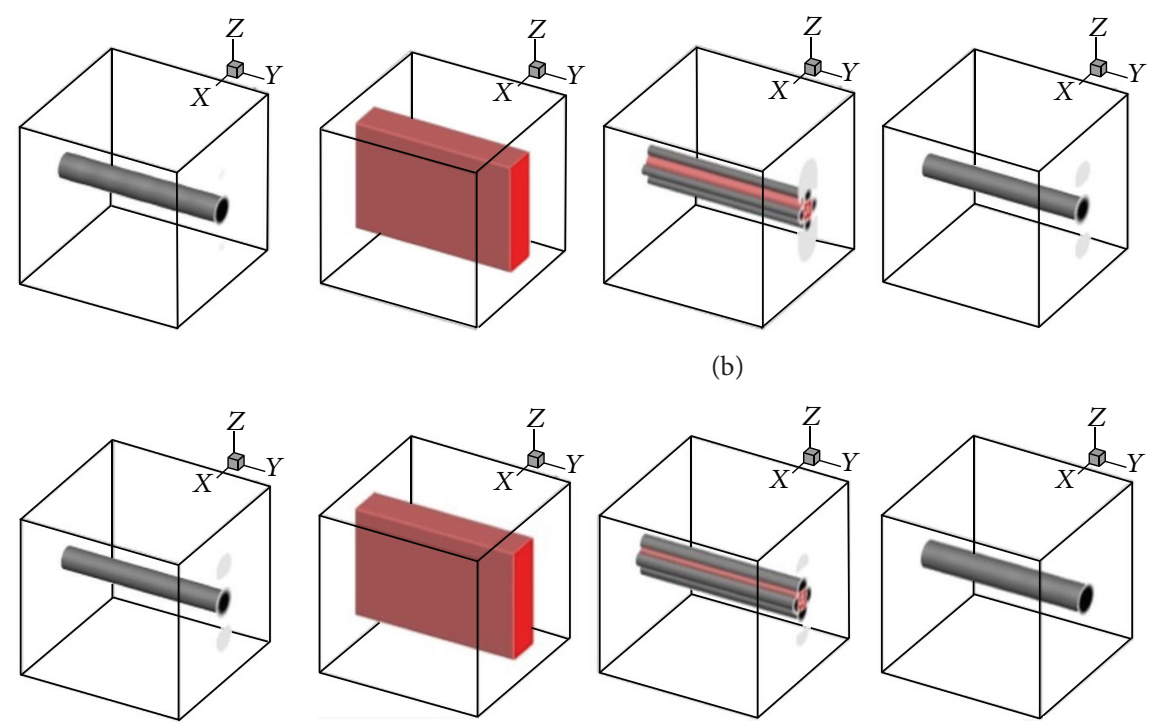

(b)
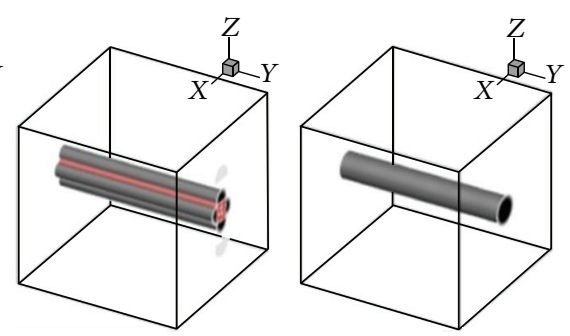

(d)
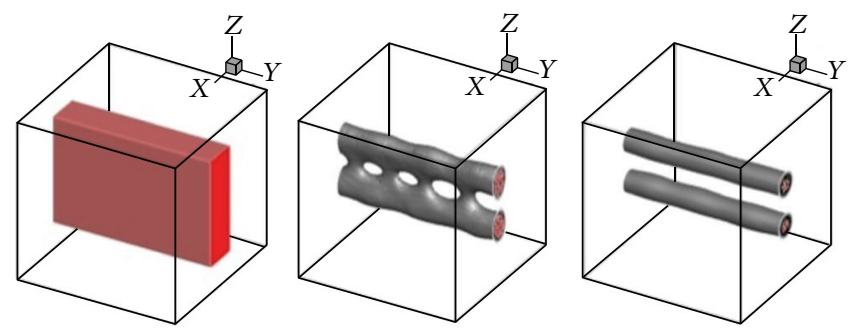

(e)

FIGURE 3: Morphological evolutions in pyrolysis with different interfacial interactions at the beginning, 5,000, and 10,000 steps: (a) $h_{11}=$ $h_{22}=h_{12}$, (b) $h_{11}>h_{22}=h_{12}$, (c) $h_{22}>h_{11}=h_{12}$, (d) $h_{11}=h_{22}>h_{12}$, and (e) $h_{11}=h_{22}<h_{12}$. 




FIgURE 4: Polymer diminutions with different reaction rates with respect to simulation time.

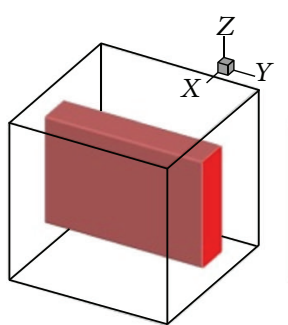

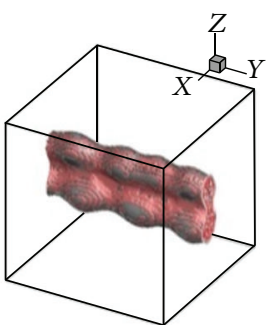

(a)

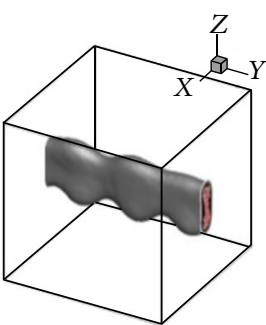

$\stackrel{1}{Z}_{X}^{Z}$
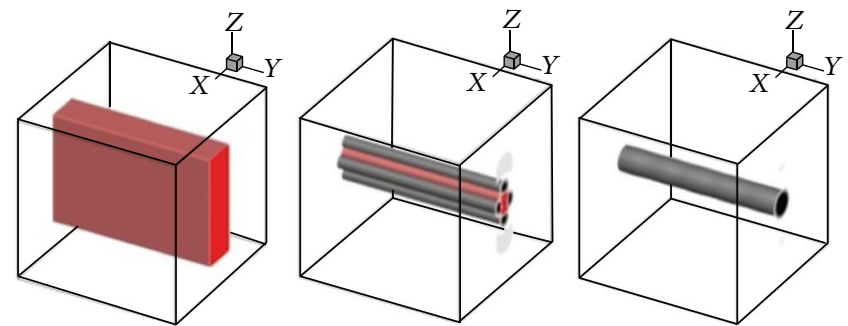

(b)
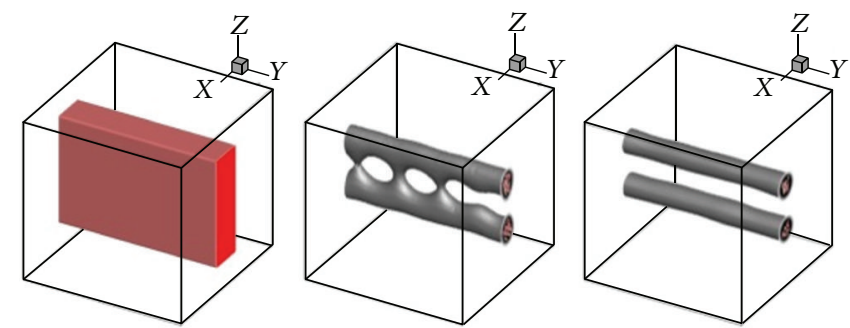

(c)

FIGURE 5: Morphological evolutions in pyrolysis with different reaction rates at the beginning, 5,000, and 10,000 steps: (a) $v=0.0003$, (b) $v=0.0005$, and (c) $v=0.0007$. All simulations are conducted with $h_{11}=h_{22}=h_{12}$.

generated as shown in Figure 3(e). When the interface energy between polymer and carbon is similar or less than the surface energies, a well-defined single structure can be obtained.

3.3. Effect of Reaction Rate. The morphological evolution proceeds with reducing the free energy of system on the diffusional time scale. In pyrolysis, the decomposition process is also related with the kinetic process. To investigate the effect of decomposition process on the morphological evolution, a series simulation is performed with different reaction rates as shown in Figures 4 and 5. The remaining polymer during pyrolysis with respect to the different reaction rates is presented in Figure 5. When the reaction rate of decomposition is very slow compared to diffusion as shown in Figure 5(a), it is difficult to obtain a well-defined structure while the diffusion process is comparable to the decomposition process. When the reaction rate of decomposition is faster than the diffusion process, multiple structures with relatively abundant carbon are generated as shown in Figure 5(c). If the balance between the decomposition rate and the diffusion process is not manipulated, the undecomposed polymer can remain inside of the generated carbon structure as shown in Figures 5(a) and $5(\mathrm{c})$ in the middle of the pyrolysis process. 


\section{Conclusions}

In this study, we developed a three-dimensional dynamic model that can simulate the morphological evolution of the decomposed polymer and generated carbon in pyrolysis. We investigated the effect of the relation of interface energies between materials and the reaction rate of decomposition on the morphological evolution during pyrolysis. Not only the structure at the early stage but also the final structures can be variously generated with manipulating the interface energies and reaction rate. The high reaction rate that may be obtained by processing conditions such as the operating temperature induces a fast decomposition process, but it may induce scattered structures rather than well-defined structures. When the interface energy between polymer and carbon is smaller than the surface energies of polymer and carbon, which can be manipulated by the different materials and preprocessing, the multiple structures also can be generated. The developed model increases the versatility of pyrolysis as a reliable nanofabrication technique by providing the structure information during the pyrolysis process.

\section{Conflict of Interests}

The authors declare that there is no conflict of interests regarding the publication of this paper.

\section{Acknowledgments}

This research was supported by the National Research Foundation of Korea (NRF) grant funded by the Ministry of Science, ICT \& Future Planning (MSIP) (nos. 20090083510, 2013R1A1A2011263, 2014R1A2A2A09052374, and 2013R1A1A1076080) and supported by the Industrial Strategic Technology Development Program (Grant no. 10041589) funded by the Ministry of Knowledge Economy (MKE, Korea).

\section{References}

[1] J. P. Diebold, A Review of the Chemical and Physical Mechanisms of the Storage Stability of Fast Pyrolysis Bio-Oils, National Renewable Energy Laboratory, Golden, Colo, USA, 2000.

[2] D. Mohan, C. U. Pittman Jr., and P. H. Steele, "Pyrolysis of wood/ biomass for bio-oil: a critical review," Energy and Fuels, vol. 20, no. 3, pp. 848-889, 2006.

[3] C. L. Wang, G. Y. Jia, L. H. Taherabadi, and M. J. Madou, "A novel method for the fabrication of high-aspect ratio C-MEMS structures," Journal of Microelectromechanical Systems, vol. 14, no. 2, pp. 348-358, 2005.

[4] K. Malladi, C. L. Wang, and M. Madou, "Fabrication of suspended carbon microstructures by e-beam writer and pyrolysis," Carbon, vol. 44, no. 13, pp. 2602-2607, 2006.

[5] B. A. Samuel, R. Rajagopalan, H. C. Foley, and M. A. Haque, "Temperature effects on electrical transport in semiconducting nanoporous carbon nanowires," Nanotechnology, vol. 19, no. 27, Article ID 275702, 2008.

[6] S. Sharma, A. Sharma, Y.-K. Cho, and M. Madou, "Increased graphitization in electrospun single suspended carbon nanowires integrated with carbon-MEMS and carbon-NEMS platforms," ACS Applied Materials and Interfaces, vol. 4, no. 1, pp. 34-39, 2012.

[7] A. G. W. Bradbury, Y. Sakai, and F. Shafizadeh, "A kinetic model for pyrolysis of cellulose," Journal of Applied Polymer Science, vol. 23, no. 11, pp. 3271-3280, 1979.

[8] F. Thurner and U. Mann, "Kinetic investigation of wood pyrolysis," Industrial \& Engineering Chemistry, Process Design and Development, vol. 20, no. 3, pp. 482-488, 1981.

[9] B. V. Babu and A. S. Chaurasia, "Parametric study of thermal and thermodynamic properties on pyrolysis of biomass in thermally thick regime," Energy Conversion and Management, vol. 45, no. 1, pp. 53-72, 2004.

[10] K. S. Ro, K. B. Cantrell, P. G. Hunt, T. F. Ducey, M. B. Vanotti, and A. A. Szogi, "Thermochemical conversion of livestock wastes: carbonization of swine solids," Bioresource Technology, vol. 100, no. 22, pp. 5466-5471, 2009.

[11] A. Broido and M. A. Nelson, "Char yield on pyrolysis of cellulose," Combustion and Flame, vol. 24, pp. 263-268, 1975.

[12] G. Várhegyi, M. J. Antal Jr., E. Jakab, and P. Szabó, "Kinetic modeling of biomass pyrolysis," Journal of Analytical and Applied Pyrolysis, vol. 42, no. 1, pp. 73-87, 1997.

[13] R. Capart, L. Khezami, and A. K. Burnham, "Assessment of various kinetic models for the pyrolysis of a microgranular cellulose," Thermochimica Acta, vol. 417, no. 1, pp. 79-89, 2004.

[14] N. Grioui, K. Halouani, A. Zoulalian, and F. Halouani, “Thermochemical modeling of isothermal carbonization of thick wood particle-effect of reactor temperature and wood particle size," Energy Conversion and Management, vol. 48, no. 3, pp. 927-936, 2007.

[15] A. Khawam and D. R. Flanagan, "Solid-state kinetic models: basics and mathematical fundamentals," The Journal of Physical Chemistry B, vol. 110, no. 35, pp. 17315-17328, 2006.

[16] M. Yang, S. Jeong, T. Kang, and D. Kim, "Equilibrium morphology of plasmonic Au/polystyrene dimeric nanoparticle," The Journal of Physical Chemistry C, vol. 119, no. 11, pp. 6148-6151, 2015.

[17] J. Song and D. Kim, "Three-dimensional chemotaxis model for a crawling neutrophil," Physical Review E, vol. 82, no. 5, Article ID 051902, 2010.

[18] D. Kim, "Computational analysis of the interfacial effect on electromigration in flip chip solder joints," Microelectronic Engineering, vol. 86, no. 10, pp. 2132-2137, 2009.

[19] J. W. Cahn and J. E. Hilliard, "Free energy of a nonuniform system. I. Interfacial free energy," Journal of Chemical Physics, vol. 28, no. 2, pp. 258-267, 1958.

[20] R. Folch and M. Plapp, "Quantitative phase-field modeling of two-phase growth," Physical Review E, vol. 72, no. 1, Article ID 011602, 2005.

[21] J. E. White, W. J. Catallo, and B. L. Legendre, "Biomass pyrolysis kinetics: a comparative critical review with relevant agricultural residue case studies," Journal of Analytical and Applied Pyrolysis, vol. 91, no. 1, pp. 1-33, 2011. 



Submit your manuscripts at http://www.hindawi.com


\section{The Scientific World Journal}
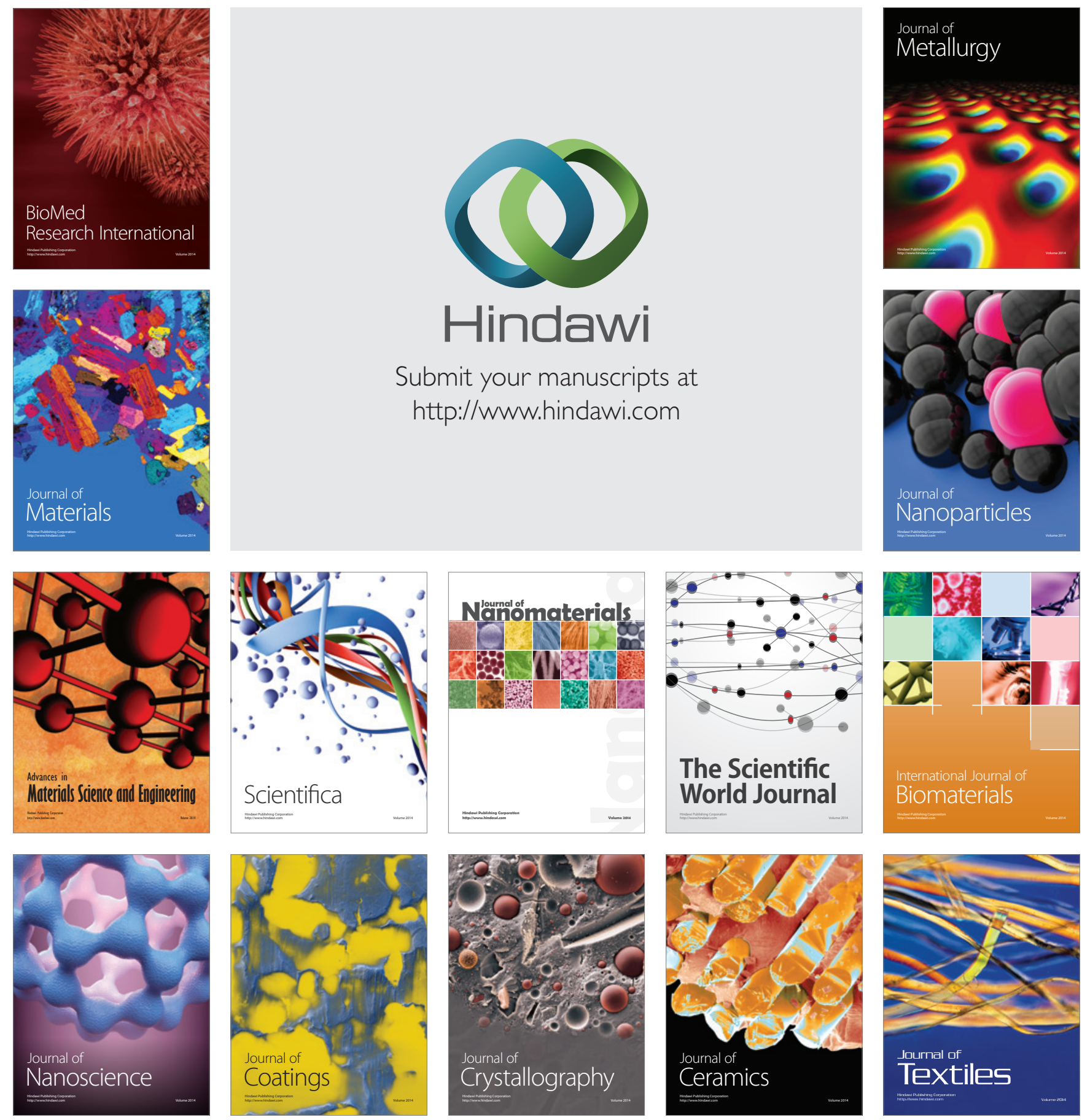